\title{
Alles nur Timing? Kommunale Wahlbeteiligung im Kontext von Bundestagswahlen und Wahlen zum Europäischen Parlament
}

\author{
Angelika Vetter
}

\section{Die Ausgangslage und das Problem}

Seit Beginn der 1990er Jahre bricht die kommunale Wahlbeteiligung in Deutschland ein. Noch Ende der achtziger Jahre lag die durchschnittliche Wahlbeteiligung in denjenigen Bundesländern, in denen in diesem Zeitraum eine Kommunalwahl stattfand, bei etwa 70 Prozent. Heute nimmt kaum mehr die Hälfte der Wahlberechtigten an den Wahlen zu den kommunalen Vertretungskörperschaften teil. Zum Beispiel in Niedersachsen und Hessen lag die Beteiligungsquote 2006 gerade noch über 50 Prozent. Im März 2008 sank sie in Bayern - einem Bundesland mit traditionell hoher Wahlbeteiligung - auf unter 60 Prozent, und rutschte im Mai desselben Jahres in Schleswig-Holstein auf 49,5 Prozent. In den neuen Bundesländern war die 50-Prozent-Marke bereits 1999 bei den Kommunalwahlen in Sachsen-Anhalt unterschritten worden. Damit sank die kommunale Wahlbeteiligung in weniger als 20 Jahren um mehr als 20 Prozentpunkte. ${ }^{1}$ Der Rückgang lässt sich nicht auf die Wiedervereinigung und die geringere Beteiligungsbereitschaft in Ostdeutschland zurückführen, denn er ist gleichermaßen in den alten und neuen Bundesländern zu beobachten.

Erkennt man an, dass auch auf lokaler Ebene relevante politische Entscheidungen getroffen werden, dann wirft diese Entwicklung Fragen nach der Legitimation und Responsivität kommunaler Entscheidungsträger auf. Wie in der nationalen Politik sind Wahlen zu den repräsentativen Vertretungskörperschaften auch hier Ausdruck der Volkssouveränität und Zeichen der demokratischen Qualität, in diesem Fall von „lokaler“ Politik. Zwar haben in den letzten Jahren gerade auf der kommunalen Ebene „neue“ Beteiligungsformen wie Referenden oder stärker dialogorientierte Verfahren an Bedeutung gewonnen. ${ }^{2}$ Sie eröffnen den Bürgern tiefergehende Mitwirkungs- beziehungsweise detailliertere Entscheidungsrechte als dies Wahlen tun und könnten aus diesem Grund die Beteiligung an Wahlen unattraktiver erscheinen lassen. Dennoch sind Wahlen diesen neuen Formen politischer Beteiligung in einigen Punkten überlegen: Erstens nehmen an ihnen noch immer weitaus mehr Bürger teil als an anderen Partizipationsformen. Zweitens ist ihr Ausgang für die politischen Entscheidungsträger verbindlich, das heißt die Gewählten müssen sich dem Votum der Bürger beugen. Drittens wird das Ergebnis der Interessenäußerung durch einen institutionalisierten und damit transparenten Mechanismus bestimmt (Wahlsystem). Viertens ist die gleichwertige Berücksichtigung der einzelnen Interessen - soweit sie geäußert werden - gesichert, da jede Stimme gleich zählt. Und fünftens erfordert die Beteiligung an Wahlen vergleichsweise wenige Ressourcen (Zeit, Geld, Wissen). Daher dürften Beteiligungsunterschiede in

1 Die kommunale Wahlbeteiligung sinkt seit den 1980er Jahren auch in vielen anderen westeuropäischen Ländern, vgl. Henk van der Kolk, Local Electoral Participation in Europe, Vortrag auf der Tagung des Arbeitskreises „Lokale Politikforschung“ der DVPW in Münster, September 2006.

2 Vgl. zum Beispiel Angelika Vetter, Lokale Bürgerbeteiligung: Ein Thema mit offenen Fragen, in: dies. (Hrsg.), Erfolgsbedingungen lokaler Bürgerbeteiligung, Wiesbaden 2008, S. 9 - 27. 
Abhängigkeit von der jeweiligen Ressourcenausstattung geringer und die geäußerten Interessen repräsentativer sein als bei anderen Formen der Partizipation. ${ }^{3}$ Die Bedeutung der Wahlen für die lokale Politik sowie die aktuelle Entwicklung der Wahlbeteiligung legen daher die Frage nahe, ob Bürger zukünftig stärker zur Beteiligung an Kommunalwahlen mobilisiert werden können, und wenn ja, wie dies geschehen kann.

In der Wahlforschung wird die Teilnahme an Wahlen durch verschiedene Faktoren erklärt. Individuelle Eigenschaften der Wähler spielen dabei ebenso eine Rolle wie institutionelle, sozialstrukturelle und politische Kontextfaktoren. ${ }^{4}$ Im vorliegenden Beitrag werden vor allem die Mobilisierungseffekte untersucht, die vom politischen Kontext der lokalen Wahlen ausgehen, genauer gesagt von ihrer zeitlichen Platzierung im Zyklus der Bundestagswahlen und von ihrer Koppelung mit Wahlen zum Europäischen Parlament (EP).

In der Literatur findet sich seit den 1960er Jahren eine zunehmend intensiver werdende Diskussion um die Zusammenhänge zwischen nationalen Haupt- und Nebenwahlen. Das zentrale Charakteristikum einer Nebenwahl (second-order election) besteht darin, dass ihr von allen Beteiligten (Wählern, Parteien, Medien) weniger Bedeutung geschenkt wird als der Hauptwahl (first-order election), weil es um weniger geht. ${ }^{5}$ Wie in vielen anderen Ländern sind in Deutschland die Wahlen zum nationalen Parlament die Hauptwahlen. Landtags-, Kommunal- und EP-Wahlen werden dagegen als Nebenwahlen angesehen. Dies belegen auch Umfragedaten, in denen die Deutschen ein signifikant geringeres Interesse an der lokalen und europäischen Politik zeigen als an der bundesdeutschen Politik. ${ }^{6}$

Im Modell der Haupt- und Nebenwahlen wird davon ausgegangen, dass sich die politische Bedeutung von Nebenwahlen mit ihrem zeitlichen Abstand von der nächsten Hauptwahl verändert. Je näher diese an der folgenden Hauptwahl liegt, desto stärker wird die Nebenwahl von der Mobilisierungswelle erfasst, die von den Parteien und den Medien angesichts der anstehenden Hauptwahl angeschoben wird. Gerade im Superwahljahr 20097

3 Vgl. Sidney Verba / Kay Lehman Schlozman / Henry E. Brady, Voice and Equality, Cambridge / London 1995.

4 Vgl. Mark N. Franklin / Cees van der Eijk / Erik Oppenhuis, The Institutional Context: Turnout, in: Cees van der Eijk / Mark N. Franklin, Choosing Europe? The European Electorate and National Politics in the Face of Union, Ann Arbor 1996, S. 306 - 331, S. 311; Angelika Vetter, Kommunale Wahlbeteiligung im Bundesländervergleich. Politische Institutionen und ihre Folgen, in: Die Öffentliche Verwaltung, 61. Jg. (2008), H. 21, S. 885 - 894.

5 Vgl. Karlheinz Reif / Hermann Schmitt, Nine Second-Order National Elections. A Conceptual Framework for the Analysis of European Results, in: European Journal of Political Research, 8. Jg. (1980), H. 1, S. 3 - 44; Karlheinz Reif, National Electoral Cycles and European Elections 1979 and 1984, in: Electoral Studies, 3. Jg. (1984), H. 3, S. $244-255$.

6 Fragestellung: „Leute interessieren sich in unterschiedlichem Maß für Politik. Wie ist das bei Ihnen: Wie stark interessieren Sie sich für (Politik hier in Ihrer Stadt/Gemeinde; Politik in Deutschland; Politik in Europa)? Sehr stark (4), ziemlich stark (3), mittelmäßig (2), weniger stark (1) oder überhaupt nicht (0)? Das Interesse an europäischer Politik liegt im Durchschnitt bei 2,16, an lokaler Politik bei 2,18 und an Politik in Deutschland bei 2,4. Quelle: Repräsentative Bevölkerungsumfrage im Auftrag der Freiherr vom Stein Akademie und des Instituts für Sozialwissenschaften der Universität Stuttgart, Januar 2008.

7 Ähnlich wie im Jahr 1994 fanden 2009 zahlreiche Wahlen statt: am 23. Mai die Wahl des Bundespräsidenten, am 7. Juni die Wahl zum Europäischen Parlament sowie Kommunalwahlen in sieben Bundesländern (Baden-Württemberg, Mecklenburg-Vorpommern, Rheinland-Pfalz, Saarland, Sachsen, Sachsen-Anhalt, Thüringen), am 30. August die Kommunalwahlen in NordrheinWestfalen sowie die Landtagswahlen im Saarland, in Sachsen, Thüringen und Brandenburg und 
stellt sich daher die Frage, inwieweit Nebenwahlen von Mobilisierungsschüben im Vorfeld von Hauptwahlen profitieren. Dies wird rückwirkend für die Kommunalwahlen in der Bundesrepublik Deutschland von 1951 bis 2008 untersucht. Ergänzend wird einer ähnlichen Logik folgend geprüft, ob durch die Zusammenlegung von Kommunal- und EP-Wahlen die Bedeutung beider Wahlen erhöht werden kann und dadurch die Beteiligungswahrscheinlichkeit und -quote steigen. Die Analysen basieren auf aggregierten Daten zu 114 Kommunalwahlen in 13 Bundesländern (ohne die Stadtstaaten Berlin, Hamburg und Bremen). ${ }^{8}$

\section{Bundestagszyklen, Koppelung und kommunale Wablbeteiligung}

Polarisierende Kandidaten, besonders wichtige Themen oder kurzfristige Ereignisse können die Wähler besonders stimulieren, sich an einer Wahl zu beteiligen. Aufgrund dieser Tatsache unterschied Angus Campbell 1960 zwischen „high -,, und „low stimulus elections“. „If the alternatives are generally seen as implying no important differences resulting from the election of one or the other, the stimulation to vote will be relatively weak. If the alternatives are seen as implying significantly different consequences, the stimulation to vote will be relatively high." Er verglich die Auswirkungen mit einer Welle, die sich bei einer high stimulus election auftürmt und bei der nächsten, weniger wichtigen Wahl wieder abflacht („surge“ und „decline“). Der unterschiedlichen Mobilisierung liegt zugrunde, dass sich nur ein Teil der Wähler überhaupt für Politik interessiert. Diese Wähler haben in der Regel eine intensive Parteibindung und nehmen, so Campbell, grundsätzlich ihr Wahlrecht wahr, unabhängig von der Bedeutung der anstehenden Wahl („core voters“). In high stimulus elections dagegen würden wegen des intensiveren Wahlkampfes der Parteien und einer umfangreicheren Medienberichterstattung neben den interessierten „core voters“ weitere Gruppen für die aktuellen politischen Themen und Kandidaten interessiert und mobilisiert. ${ }^{10}$

Wahlen mit hohem und geringerem Stimulus differieren jedoch nicht nur hinsichtlich der Beteiligung. In dem Maße, in dem sich interessierte und weniger interessierte Wähler in ihren politischen Präferenzen unterscheiden, kann die Veränderung der Wahlbeteiligung auch mit einer Veränderung der politischen Mehrheitsverhältnisse einhergehen. Dies zeigt sich in den USA vor allem beim Vergleich der Präsidentschaftswahlen - den high stimulus elections - mit den folgenden „low stimulus“ beziehungsweise Midterm-Kongresswahlen,

am 27. September die Wahl des Brandenburgischen Landtags, die zeitlich mit der Bundestagswahl stattfand.

8 Ich danke an dieser Stelle besonders Elisa Helbig für ihre Hilfe bei der Recherche und Aufbereitung der Daten. Dabei wurde zurückgegriffen auf die Homepages der Statistischen Landesämter, auf http://www.sgk-online.net/, sowie auf Verband Deutscher Städtestatistiker (Hrsg.), Politische Wahlen in 65 Großstädten und in Bundesländern 1949-1987, Duisburg 1987. Außerdem danke ich Jürgen Maier und Frank Brettschneider für ihre hilfreichen Kommentare sowie der DFG für die finanzielle Unterstützung des Projektes „Wahlbeteiligung in den Städten und Gemeinden der Bundesrepublik“.

9 Angus Campbell, Surge and Decline: A Study of Electoral Change, in: Public Opinion Quarterly, 24. Jg. (1960), H. 3, S. $397-418$, S. 398.

10 Vgl. ebenda, S. 400. 
bei denen die Wahlbeteiligung wieder auf das erwartbar „normale“ Niveau absinkt und die Partei des Präsidenten in der Regel einen deutlichen Stimmenverlust erleidet. ${ }^{11}$

Mit der ersten Wahl des Europäischen Parlaments 1979 tauchen in der europäischen Wahlforschung zum ersten Mal die Begriffe der „Haupt-“ und „Nebenwahlen“ beziehungsweise der „first-“ und „second-order elections“ auf. ${ }^{12}$ Karlheinz Reif und Hermann Schmitt untersuchten mit diesem Konzept die Abhängigkeit der EP-Wahlergebnisse (Wahlbeteiligung und Stimmenverteilung) von den Konstellationen in den jeweiligen nationalen politischen Arenen. Ähnlich wie die US-amerikanische Wahlforschung gingen sie davon aus, dass die Wähler zwischen Haupt- und Nebenwahlen unterscheiden, weil sie ihnen verschiedene Bedeutung zumessen, weil bei ihnen einmal mehr und einmal weniger ,auf dem Spiel steht“. Diese unterschiedliche Bedeutungszuschreibung hat nach Reif und Schmitt folgende Konsequenzen: (1) die Wahlbeteiligung bei Nebenwahlen ist niedriger als bei Hauptwahlen; (2) der Anteil ungültiger Stimmen ist bei Nebenwahlen höher als bei Hauptwahlen; (3) kleinere und neuere Parteien haben bei Nebenwahlen bessere Chancen als bei Hauptwahlen; (4) die regierenden Parteien bekommen bei Nebenwahlen weniger Stimmen als bei Hauptwahlen. ${ }^{13}$

Im Unterschied zu US-amerikanischen Kollegen ging Reif jedoch davon aus, dass sich die genannten Unterschiede in der Stimmverteilung zwischen Haupt- und Nebenwahlen verändern, und zwar je nach Zeitpunkt im nationalen Wahlzyklus. „... the time that has passed since the last FOE (first-order election) is the crucial variable structuring SOE (second-order election) results. " 14 Je näher die Nebenwahlen an einer Hauptwahl liegen, desto ähnlicher sind die Stimmverteilungen. Je weiter die Nebenwahl dagegen zeitlich von einer Hauptwahl entfernt liegt (Maximum in der Mitte der Wahlperiode), desto stärker weicht die Stimmverteilung von den Ergebnissen der letzten Hauptwahl ab. Hinsichtlich der Wahlbeteiligung vermutete er allerdings nur, dass Unterschiede zur Hauptwahl je nach Grad ihrer „Unwichtigkeit“ bestehen. „Turnout at SOE, regularly lower than FOE turnout, reflects the relevance and weight of different SOE, i.e. reflects how much there is perceived to be at stake." 15 Dass sich auch die Wahlbeteiligung mit der Positionierung der Nebenwahl im Hauptwahlzyklus verändern könnte, wurde nicht weiter verfolgt.

Diese Überlegung tauchte erst später auf. Für die Beteiligung an Europawahlen zeigen Franklin, van der Eijk und Oppenhuis, dass die Beteiligung umso höher ausfällt, je näher sie zeitlich an der folgenden nationalen Hauptwahl liegt. ${ }^{16}$ Möglicherweise, so die Autoren,

11 Mit diesen Effekten, vor allem den Stimmenveränderungen zwischen den Präsidentschafts- und Midterm-Kongresswahlen, beschäftigten sind zahlreiche Arbeiten, zum Beispiel Edward R. Tufte, Determinants of the Outcomes of Midterm Congressional Elections, in: American Political Science Review, 69. Jg. (1975), H. 4, S. 812 - 826; Michael Lewis-Beck / Tom Rice, Forecasting U.S. House Elections, in: Legislative Studies Quarterly, 9. Jg. (1984), H. 3, S. 475 - 486; Samuel Kernell, Presidential Popularity and Negative Voting: An Alternative Explanation of the Midterm Congressional Decline of the President's Party, in: American Political Science Review, 71. Jg. (1977), H. 1, S. 44 - 66; James E. Campbell, The Revised Theory of Surge and Decline, in: American Journal of Political Science, 31. Jg. (1987), H. 4, S. 965 - 979.

12 Karlheinz Reifl Hermann Schmitt, a.a.O. (Fn. 5); Karlheinz Reif, a.a.O. (Fn. 5).

13 Vgl. Karlheinz Reif/ Hermann Schmitt, a.a.O. (Fn. 5), S. 9.

14 Karlheinz Reif, a.a.O. (Fn. 5), S. 246.

15 Ebenda, S. 247.

16 Mark N. Franklin / Cees van der Eijk / Erik Oppenhuis, a.a.O. (Fn. 4), S. 306; ähnlich Michael Marsh / Mark N. Franklin, The Foundations: Unanswered Questions from the Study of European 
werden Europawahlen kurz vor einer nationalen Hauptwahl von der jeweiligen Mobilisierungswelle erfasst, die eine Folge der größeren Anstrengung der nationalen Parteien, der größeren Medienaufmerksamkeit und einem damit verbundenen Anstieg des Interesses in der Bevölkerung ist. Außerdem kamen sie zu dem Ergebnis, dass es sich hierbei um einen linearen Effekt handelt. Das heißt, die Wahlbeteiligung ist nach einer Hauptwahl eher gering, steigt vor der nächsten nationalen Hauptwahl aber an. ${ }^{17}$

Für Deutschland liegen bislang lediglich Studien vor, die den Zusammenhang zwischen dem Bundestagswahlzyklus und den Ergebnissen bei Landtagswahlen untersuchen. Ein bundespolitischer Einfluss auf die Wahlentscheidung bei Landtagswahlen ist dabei weitgehend unumstritten. Die Studien belegen, dass die im Bund regierenden Parteien bei den Landtagswahlen in der Regel mit Stimmenverlusten rechnen müssen. Zumindest für die Zeit vor 1990 veränderten sich diese zyklisch mit der Entfernung zur Hauptwahl. Je weiter der Abstand zur letzten und zur nächsten Hauptwahl ist, desto schlechter schnitten die Regierungsparteien im Bund ab (Maximum in der Mitte der Wahlperiode). Jedoch bestehen unterschiedliche Ansichten über die Stärke des Einflusses, die Ursachen und die Entwicklung nach $1990 .{ }^{18}$

Für Kommunalwahlen gibt es hierzu bislang überhaupt keine Untersuchungen. Aus repräsentativen Bevölkerungsumfragen, die seit 1979 durchgeführt wurden, lässt sich für die letzten 30 Jahre belegen, dass die Bürger die Entscheidungen der Kommunen und Landesparlamente für deutlich weniger wichtig halten als die vom Deutschen Bundestag getroffenen (vgl. Abbildung 1). Der Nebenwahl-Charakter von Kommunalwahlen ist damit für West- wie für Ostdeutschland gleichermaßen nachgewiesen. Inwieweit dies Folgen für die kommunale Wahlbeteiligung hat, ist jedoch unklar. Einerseits müsste das Haupt- und Ne-

Elections, 1979-1994, in: Cees van der Eijk / Mark N. Franklin (Hrsg.), a.a.O. (Fn. 4), S. 11 32, S. 19; Richard S. Flickinger / Donley T. Studlar, One Europe, Many Electorates? Models of Turnout in European Parliament Elections After 2004, in: Comparative Political Studies, 40. Jg. (2007), H. 4, S. $383-404$.

17 Vgl. Mark N. Franklin / Cees van der Eijk / Erik Oppenhuis, a.a.O. (Fn. 4), S. 317, Fn. 21.

18 Vgl. Reiner Dinkel, Der Zusammenhang zwischen Bundes- und Landtagswahlergebnissen, in: PVS, 18. Jg. (1977), H. 2/3, S. 348 - 359; Charlie Jeffery / Daniel Hough, The Electoral Cycle and Multi-Level Voting in Germany, in: German Politics, 10. Jg. (2001), H. 2, S. 73 - 98; Frank Decker / Julia von Blumenthal, Die bundespolitische Durchdringung der Landeswahlen. Eine empirische Analyse von 1970 bis 2001, in: ZParl, 33. Jg. (2002), H. 1, S. 144 - 165; Charlie Jeffery / Daniel Hough, Landtagswahlen: Bundestestwahlen oder Regionalwahlen?, in: Zeitschrift für Parlamentsfragen, 34. Jg. (2003), H. 1, S. 79 - 94; Simone Burkhart, Parteipolitikverflechtung - Der Einfluss der Bundespolitik auf Landtagswahlentscheidungen von 1976 bis 2002, in: PVS, 46. Jg. (2005), S. 14 - 38; Klaus Detterbeck, Zusammenlegung von Bundes- und Landtagswahlen? Die Terminierung von Wahlen und ihre Konsequenzen im europäischen Vergleich, Gütersloh 2006. Vgl. auch das Schwerpunktthema in der ZParl, 38. Jg. (2007), H. 3: insbesondere Oscar W. Gabriel I Everhard Holtmann, Ober sticht Unter? Zum Einfluss der Bundespolitik auf Landtagswahlen: Kontext, theoretischer Rahmen und Analysemodelle, S. 445 - 462; Kai-Uwe Schnapp, Landtagswahlen und Bundespolitik: immer noch eine offene Frage? Neue Antworten im Ländervergleich auf Aggregatdatenbasis, S. 463 - 480; Kerstin Völkl, Welchen Einfluss hat die Bundespolitik auf die Wahlentscheidung der Bürger bei Landtagswahlen? Eine Analyse von Individualdaten im Bundesländer- und Zeitvergleich, S. 480 - 491. Vgl. dies., Reine Landtagswahlen oder regionale Bundestagswahlen? Eine Untersuchung der Bedeutung des relativen Gewichts bundes- und landespolitischer Faktoren auf das Abstimmungsverhalten bei Landtagswahlen, Dissertation am Institut für Sozialwissenschaften der Universität Stuttgart, Stuttgart 2008. 


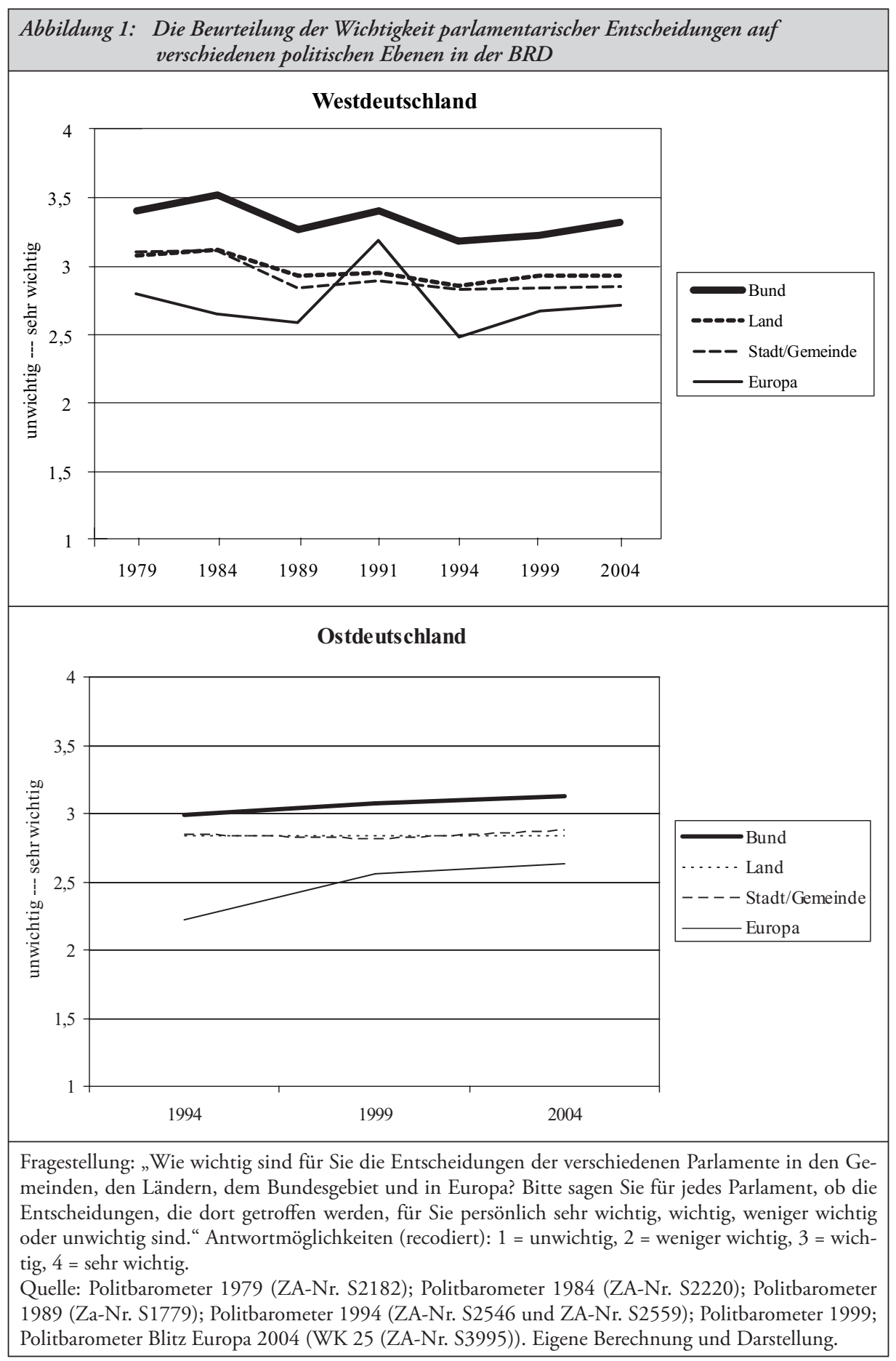


benwahl-Modell auch auf Kommunalwahlen übertragbar sein. Sie dürften damit ähnlichen Zyklusregeln unterliegen wie andere subnationale Wahlen. Andererseits könnte sich der bundespolitische Einfluss auf die Stimmenverteilung aufgrund des stärker personenbezogenen Wahlrechts und der Bedeutung der Wählergemeinschaften auf der lokalen Ebene abschwächen. Hinzu kommt, dass die Kommunalwahlen weder an einem bundeseinheitlichen Termin stattfinden, noch für die Bundespolitik relevant sind. Beide Aspekte haben zur Folge, dass sowohl die Parteien als auch die Medien den Kommunalwahlen geringere Aufmerksamkeit schenken als den Landtagswahlen, deren Ergebnisse für die Zusammensetzung des Bundesrates und damit für die Regierungspolitik im Bund von Bedeutung sind. Bundespolitische Einflüsse auf die Stimmenverteilung sind folglich bei Kommunalwahlen weniger zu erwarten als bei Landtagswahlen. Diese Überlegungen gelten jedoch nicht unbedingt für Einflüsse auf die Höhe der lokalen Wahlbeteiligung. Vielmehr kann folgende Hypothese aufgestellt werden: Je näher eine Kommunalwahl am Wahltermin der folgenden Bundestagswahl liegt, desto höher ist die Wahlbeteiligung (lineare Haupt-NebenwahlThese).

Die verstärkte Mobilisierung bei der Kommunalwahl ergibt sich erstens aus einer insgesamt gestiegenen Politisierung der Gesellschaft durch die Wahlkampagnen der Bundesparteien und die häufigere Berichterstattung der Medien. Zweitens dürfte auch die Wahlkampfarbeit vor Ort angesichts der anstehenden Hauptwahl bereits bei den Nebenwahlen intensiver ausfallen, so dass mit einer höheren Mobilisierung zu rechnen ist. Sowohl die Parteianhänger (Stammwähler) als auch „periphere“ Wähler (in der Regel ohne Parteibindung), die normalerweise kaum an einer Kommunalwahl teilnehmen, müssten im Umfeld einer Bundestagswahl damit eher zur Stimmabgabe bereit sein als in der Mitte der Wahlperiode.

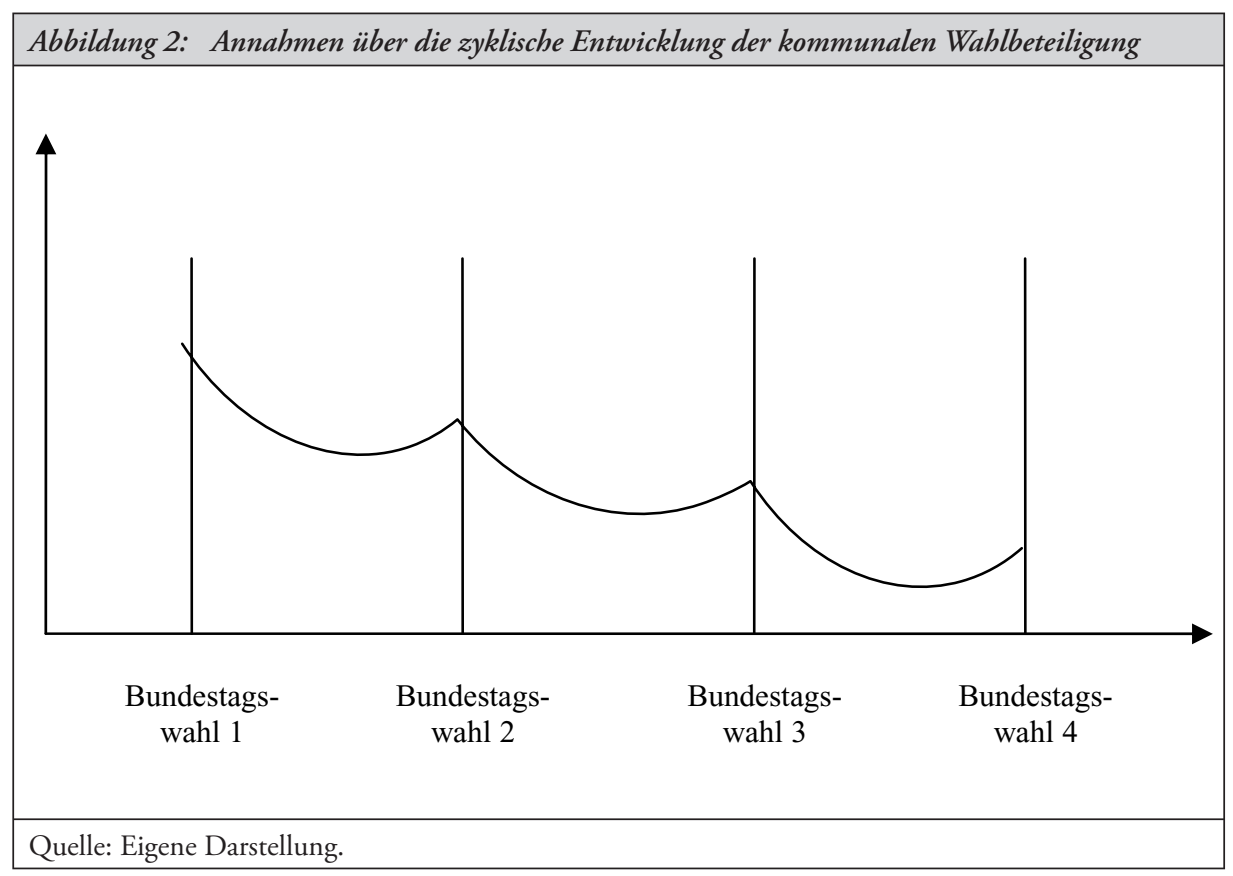


Alternativ hierzu ist allerdings möglich, dass sich die Höhe der kommunalen Wahlbeteiligung - ähnlich wie die Stimmenverteilung bei Landtagswahlen - in Abhängigkeit vom jeweiligen Bundeswahlzyklus zyklisch verändert. Dies wäre dann der Fall, wenn die Mobilisierungswelle über den eigentlichen Bundestagswahltermin hinweg anhält und erst langsam abebbt. Ihren Tiefpunkt erreicht sie dann etwa zwischen zwei Bundestagswahlen, um mit Beginn des neuen Wahlkampfes allmählich wieder anzusteigen (vgl. Abbildung 2). Die alternativ zu prüfende Hypothese lautet demnach: Je näher eine Kommunalwahl an einer Bundestagswahl liegt, desto höher ist die Wahlbeteiligung. In der Mitte der Wahlperiode ist sie am geringsten (zyklische Haupt-Nebenwahl-These).

Unabhängig von der Art der Beziehung zwischen dem Bundestagswahltermin und der kommunalen Wahlbeteiligung sind jedoch auch Veränderungen dieser Beziehung über die letzten Jahrzehnte hinweg denkbar. Stärkere Zykluseffekte ab Anfang/Mitte der 1980er Jahre wären aus zwei Gründen plausibel. Die Wahlbeteiligung hängt unter anderem vom Vorhandensein einer individuellen Parteibindung ab, das heißt, Wähler mit einer solchen Bindung gehen eher zur Wahl als Bürger ohne. ${ }^{19}$ Die Parteiidentifikation hat in der Bundesrepublik Deutschland seit den 1980er Jahren jedoch deutlich abgenommen. Daher dürfte ein Großteil der potenziellen Wähler heute stärker von externen Faktoren zur Wahl mobilisiert werden als in früheren Jahrzehnten. Zweitens können politisch wenig interessierte Bürger heute viel leichter über das Fernsehen erreicht werden, weil dieses seit den 1980er Jahren nahezu flächendeckend in allen deutschen Haushalten vorhanden ist. Auch dies dürfte heutzutage zu einer stärkeren Mobilisierung der Bevölkerung in Bundestagswahlzeiten beigetragen haben, die auf die Nebenwahlen ausstrahlt und dort die Beteiligung erhöht. Die dritte zu prüfende Annahme lautet folglich: Die Mobilisierungseffekte durch die Nähe des Nebenwahl-Termins zur Hauptwahl sind heute (seit den 1980er Jahren) stärker als in den Jahrzehnten zuvor.

Schließlich könnten sich die Mobilisierungseffekte durch die anstehende Bundestagswahl auch in Abhängigkeit vom bereits vorliegenden Mobilisierungsniveau verändern. Wenn dieses bei Kommunalwahlen auf Grund unterschiedlicher Faktoren (zum Beispiel institutionelle, sozial-strukturelle oder kulturelle Aspekte) bereits hoch ist, dann dürften „externe“ Bundestags- oder EP-Wahleffekte weniger zur Wirkung kommen. Je nach Art der vorherrschenden Politischen Kultur, hier verstanden als die traditionell in einem Bundesland vorliegende lokale Beteiligungsbereitschaft, könnten sich also verschiedene Bundestagswahl- und Koppelungseffekte ergeben. Die institutionellen Rahmenbedingungen kommunaler Politik unterscheiden sich von Bundesland zu Bundesland deutlich voneinander, zum Beispiel im Hinblick auf die verwendeten Wahlsysteme oder die Kompetenzverteilung zwischen Bürgermeistern und Räten. Obwohl es in den letzten Jahren zu einer Angleichung gekommen ist, sind die Unterschiede noch klar erkennbar. ${ }^{20}$ Ebenso gibt es - wie später gezeigt werden wird - deutliche Beteiligungsunterschiede zwischen den Bundesländern. Durch den Bundestagswahlkampf induzierte Mobilisierungseffekte dürften folglich dort besonders gut zu sehen sein, wo traditionell eine eher geringe Beteiligungsbereitschaft vorliegt.

19 Vgl. Klaus Armingeon, Gründe und Folgen geringer Wahlbeteiligung, in: Kölner Zeitschrift für Soziologie und Sozialpsychologie, 46. Jg. (1994), H. 1, S. $43-64$.

20 Vgl. Angelika Vetter, Citizens versus Parties: Explaining Institutional Change in German Local Government 1989-2008, in: Local Government Studies, 35. Jg. (2009), H. 1, S. 125 - 142. 
Der Mobilisierungslogik folgend wird abschließend geprüft, welche Folgen die Koppelung verschiedener Wahlen für die Höhe der Wahlbeteiligung hat, da auch dies die politische Bedeutung einer Wahl verändern kann. Bislang fanden in der Bundesrepublik Deutschland drei Kommunalwahlen zeitgleich mit einer Bundestagswahl statt und einmal am selben Tag mit einer Landtagswahl. ${ }^{21}$ In allen vier Fällen kam es zu signifikanten „Ausreißern“ in der kommunalen Wahlbeteiligung, die deutlich über dem erwarteten Wert lagen. In einigen Bundesländern werden Kommunalwahlen schon seit geraumer Zeit gemeinsam mit den Wahlen zum EP durchgeführt (vgl. Tabelle 1 und Abbildung 3). Ein Argument für diese Koppelung der Wahlen sind die geringen Kosten einer gemeinsamen Wahl. Gleichzeitig aber steht dahinter die Erwartung, die Attraktivität des Wahlgangs (es ist über mehr zu entscheiden als bei nur einer Wahl) zu erhöhen bei gleichzeitiger Senkung des Aufwands für den einzelnen Bürger. Beides müsste sich theoretisch positiv in der Wahlbeteiligung niederschlagen. Die Realität hat allerdings gezeigt, dass sich in der Regel noch weniger Bürger an der EP-Wahl beteiligen als an Kommunalwahlen. Die Zusammenlegung von zwei „unattraktiven“ Wahlen könnte demnach entgegen der Koppelungsannahme auch folgenlos bleiben. Die Folgen der Wahlkoppelung wurden bislang nicht systematisch untersucht und sollen hier als fünfte Hypothese formuliert werden: Wenn Kommunal- und EP-Wahlen am selben Tag stattfinden, ist die Wahlbeteiligung auf Grund einer höheren Attraktivität der Wahl (,it is more at stake“) überdurchschnittlich hoch.

\section{Kommunale Wahlbeteiligung in Deutschland}

Zwischen 1949 und 1989 veränderte sich die kommunale Wahlbeteiligung in Deutschland kaum. Zur Zeit der ersten Wahlperiode des Bundestages (1949 bis 1953) lag sie im Durchschnitt bei 76,7 Prozent. In den 1970er Jahren lassen sich einige Ausreißer erkennen, die auf Zusammenlegungen von kommunalen Wahlen mit Bundestags- oder Landtagswahlen zurückzuführen sind. Zu dieser Zeit betrug die kommunale Wahlbeteiligung im Durchschnitt der Bundesländer fast 80 Prozent (inklusive Ausreißer), und während der elften Wahlperiode des Bundestages (1987 bis 1990) lag der Durchschnittswert noch immer bei 72,3 Prozent. Mitte der 1990er Jahre setzte jedoch ein deutlicher Rückgang der kommunalen Wahlbeteiligung ein, der bis heute anhält. Er lässt sich für alle Bundesländer feststellen, egal ob in Ost- oder Westdeutschland, und beträgt für die zwanzig Jahre von 1987 bis 2008 im Durchschnitt etwa 20 Prozentpunkte (vgl. Tabelle 1).

Neben dem generellen Rückgang der kommunalen Wahlbeteiligung zeigen die Daten auch deutliche und dauerhafte Niveauunterschiede zwischen den Bundesländern. Seit der Gründung der Bundesrepublik Deutschland ist die Beteiligung an kommunalen Wahlen in Baden-Württemberg durchweg am niedrigsten. Vergleichsweise hoch war sie schon immer im Saarland, aber auch in Bayern, Hessen und Rheinland-Pfalz. Die Beteiligungsquoten in Niedersachsen, Schleswig-Holstein und Nordrhein-Westfalen lagen meist im Mittelfeld. Die genannten Differenzen im Beteiligungsniveau zwischen den Bundesländern betragen bis zu 20 Prozentpunkte. Dies legt die Vermutung nahe, dass die unterschiedlichen institu-

21 Zeitgleich mit Bundestagswahlen fanden Kommunalwahlen 1976 in Niedersachsen, 1995 in Nordrhein-Westfalen und 1998 in Brandenburg statt. Die Kommunalwahl 1975 in NordrheinWestfalen lag auf demselben Tag wie die dortige Landtagswahl. 


\begin{tabular}{|c|c|c|c|c|c|c|c|}
\hline \multicolumn{8}{|c|}{$\begin{array}{l}\text { Tabelle 1: Kommunale Wablbeteiligung nach Bundesländern und Wahlperioden des Bundestages, } \\
1987 \text { bis } 2008\end{array}$} \\
\hline \multirow[b]{3}{*}{ Bundesland } & \multicolumn{6}{|c|}{ Legislaturperiode / Jahre } & \multirow{3}{*}{$\begin{array}{c}\text { Differenz } \\
1987-2008 \\
\text { in } \\
\text { Prozent- } \\
\text { punkten }\end{array}$} \\
\hline & 11. & 12. & 13. & 14. & 15. & 16. & \\
\hline & $\begin{array}{l}1987- \\
1990\end{array}$ & $\begin{array}{l}1990- \\
1994\end{array}$ & $\begin{array}{l}1994- \\
1998\end{array}$ & $\begin{array}{l}1998- \\
2002\end{array}$ & $\begin{array}{l}2002- \\
2005\end{array}$ & $\begin{array}{l}2005- \\
2008\end{array}$ & \\
\hline Schleswig-Holstein $(\mathrm{SH})$ & - & 69,4 & 70,5 & 62,8 & 54,4 & 49,5 & $-19,9$ \\
\hline Niedersachsen (NS) & - & 68,3 & 64,5 & 56,2 & - & 51,8 & $-16,5$ \\
\hline Nordrhein-Westfalen (NW) & 65,6 & - & $81,7^{\mathrm{a}}$ & 55,0 & 54,5 & - & $-11,1$ \\
\hline Hessen (HE) & 78,0 & 71,3 & 66,0 & 52,9 & - & 45,8 & $-32,2$ \\
\hline Rheinland-Pfalz (RP) & $77,2^{\mathrm{b}}$ & - & $74,1^{b}$ & $62,9^{\mathrm{b}}$ & 57,6 & - & $-19,6$ \\
\hline Baden-Württemberg (BW) & 61,4 & - & $66,7^{\mathrm{b}}$ & 53,0 & $52,0^{\mathrm{b}}$ & - & $-9,7$ \\
\hline Bayern (BY) & - & 75,0 & 67,3 & - & 63,2 & 59,6 & $-15,4$ \\
\hline Saarland (SL) & $79,1^{\mathrm{b}}$ & - & $73,9^{\mathrm{b}}$ & $59,3^{\mathrm{b}}$ & $56,3^{\mathrm{b}}$ & - & $-22,8$ \\
\hline Brandenburg (BB) & - & 59,7 & - & $77,9^{\mathrm{a}}$ & 46,0 & - & $-13,7$ \\
\hline Mecklenburg-Vorp. (MV) & - & - & $65,7^{\mathrm{b}}$ & $50,5^{\mathrm{b}}$ & $44,9^{\mathrm{b}}$ & - & $-20,8$ \\
\hline Sachsen $(\mathrm{SN})$ & - & - & $70,1^{b}$ & $53,8^{\mathrm{b}}$ & $46,1^{\mathrm{b}}$ & - & $-24,0$ \\
\hline Sachsen-Anhalt (SA) & - & - & $66,2^{\mathrm{b}}$ & $49,6^{\mathrm{b}}$ & $42,1^{\mathrm{b}}$ & - & $-24,1$ \\
\hline Thüringen $(\mathrm{TH})$ & - & - & $72,3^{\mathrm{b}}$ & $58,1^{\mathrm{b}}$ & 50,6 & - & $-21,7$ \\
\hline Mittelwert* & 72,3 & 68,7 & 69,9 & 57,7 & 51,6 & 51,6 & $-20,7$ \\
\hline Nationale Wahlbeteiligung & 84,3 & 77,8 & 79,0 & 82,2 & 79,1 & 77,7 & $-0,1$ \\
\hline \multicolumn{8}{|c|}{$\begin{array}{l}\text { * Die Durchschnittswerte basieren auf den Beteiligungsmittelwerten aus denjenigen Bundesländern, in } \\
\text { denen in der genannten Wahlperiode eine Kommunalwahl stattgefunden hat. Gewichtete Mittelwerte } \\
\text { (nach der Zahl der Kommunen pro Bundesland) führen nur zu geringfügigen Abweichungen. } \\
\text { a Kommunalwahlen zeitgleich mit den Bundestagswahlen } 1994 \text { und 1998. } \\
\text { b Kommunalwahlen zeitgleich mit Wahlen zum Europäischen Parlament 1989, 1994, } 1999 \text { und } 2004 . \\
\text { Quelle: Verband Deutscher Städtestatistiker (Hrsg.), a.a.O. (Fn. 8), Ausgabe 2; eigene Recherchen auf } \\
\text { den Seiten der statistischen Landesämter; http://www.sgk-online.net (Abruf im Juli 2009). }\end{array}$} \\
\hline
\end{tabular}

tionellen Kontextbedingungen das Beteiligungsniveau beeinflussen. Bisherige Analysen hierzu bestätigen solche Effekte jedoch nicht. ${ }^{22}$ Sie sind an dieser Stelle auch nicht von Bedeutung. Zentral im Rahmen dieses Beitrags sind vielmehr Beteiligungsunterschiede zwischen den verschiedenen Wahlen (vgl. Abbildung 3). Vergleicht man pro Bundesland beispielsweise die Beteiligung der Bürger an den Kommunalwahlen mit der Beteiligung an Wahlen zum Bundestag, zu den jeweiligen Landtagen und zum EP, zeigt sich ein relativ einheitliches Muster. ${ }^{23}$ An den Bundestagswahlen - den Hauptwahlen - nehmen überall die meisten Bürger teil. Nahezu ausnahmslos folgen heutzutage die Landtagswahlen an zweiter Stelle, gemessen an der Höhe der Wahlbeteiligung. In einigen Bundesländern lag die kommunale Wahlbeteiligung über viele Jahre hinweg etwa gleichauf mit der Beteiligung an Landtagswahlen. Heute scheint hier teilweise eine Entkoppelung stattgefunden zu haben, die Landtagswahlen zumindest hinsichtlich der Wahlbeteiligung den Rang von „second-order elections“ zuschreibt, Kommunalwahlen dagegen zu „third-order elections“

23 In den hier nicht dargestellten Bundesländern sind die Muster ähnlich. 

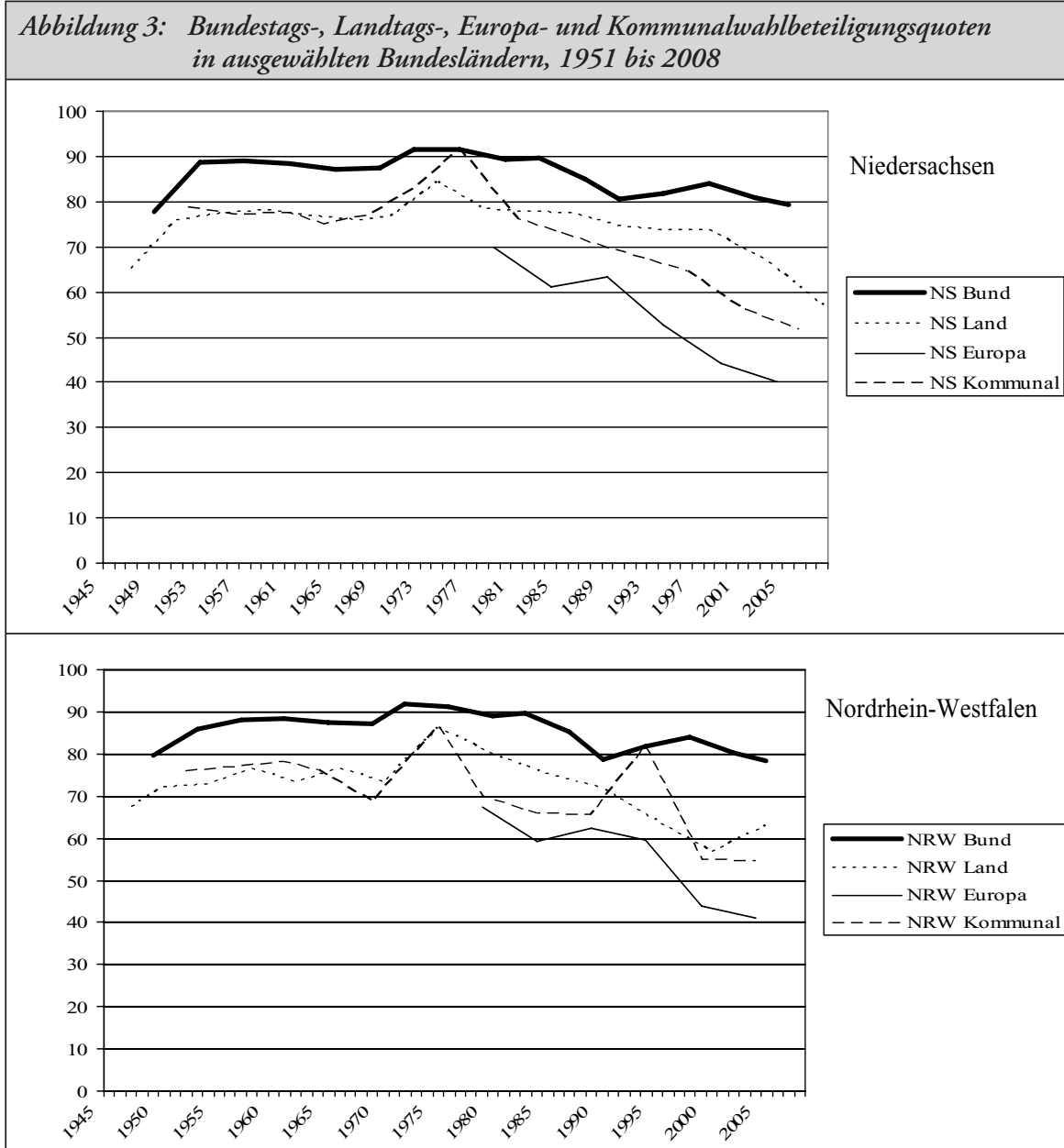

Nordrhein-Westfalen
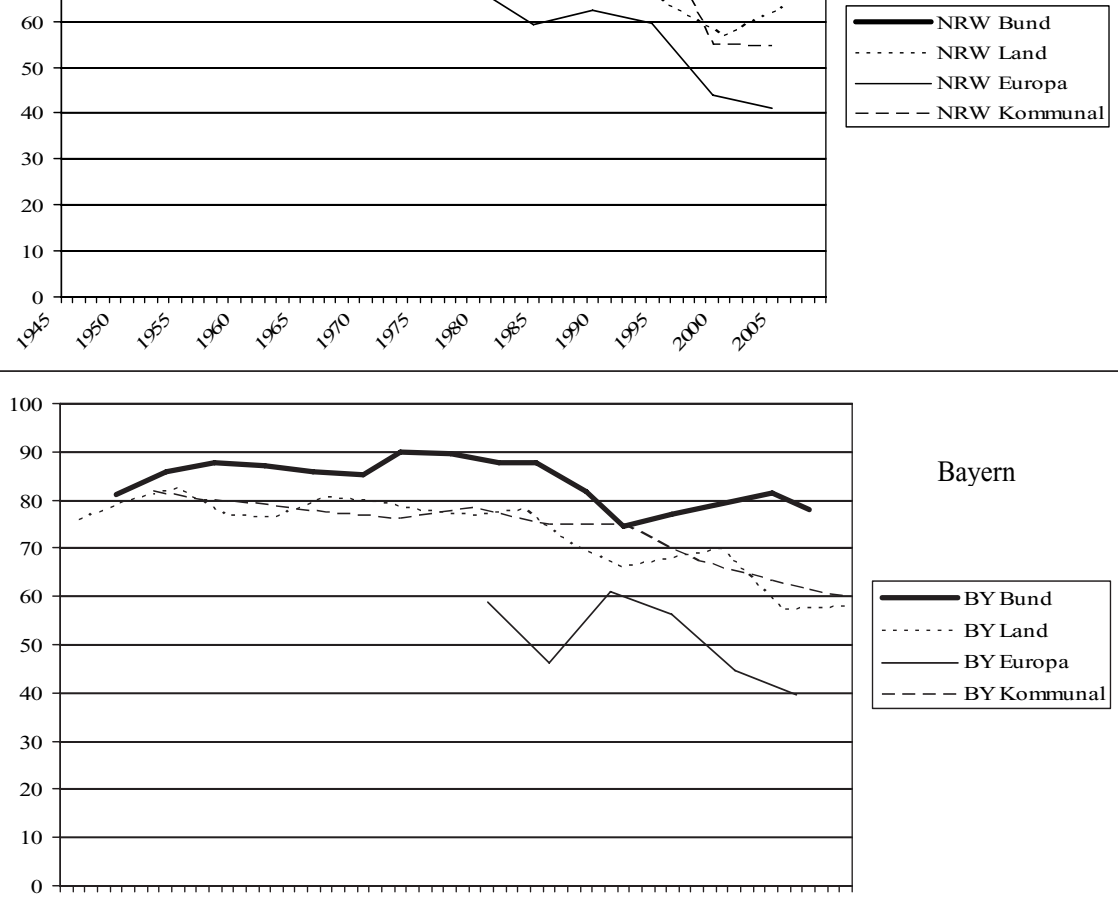

Bayern

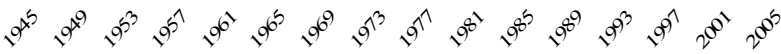




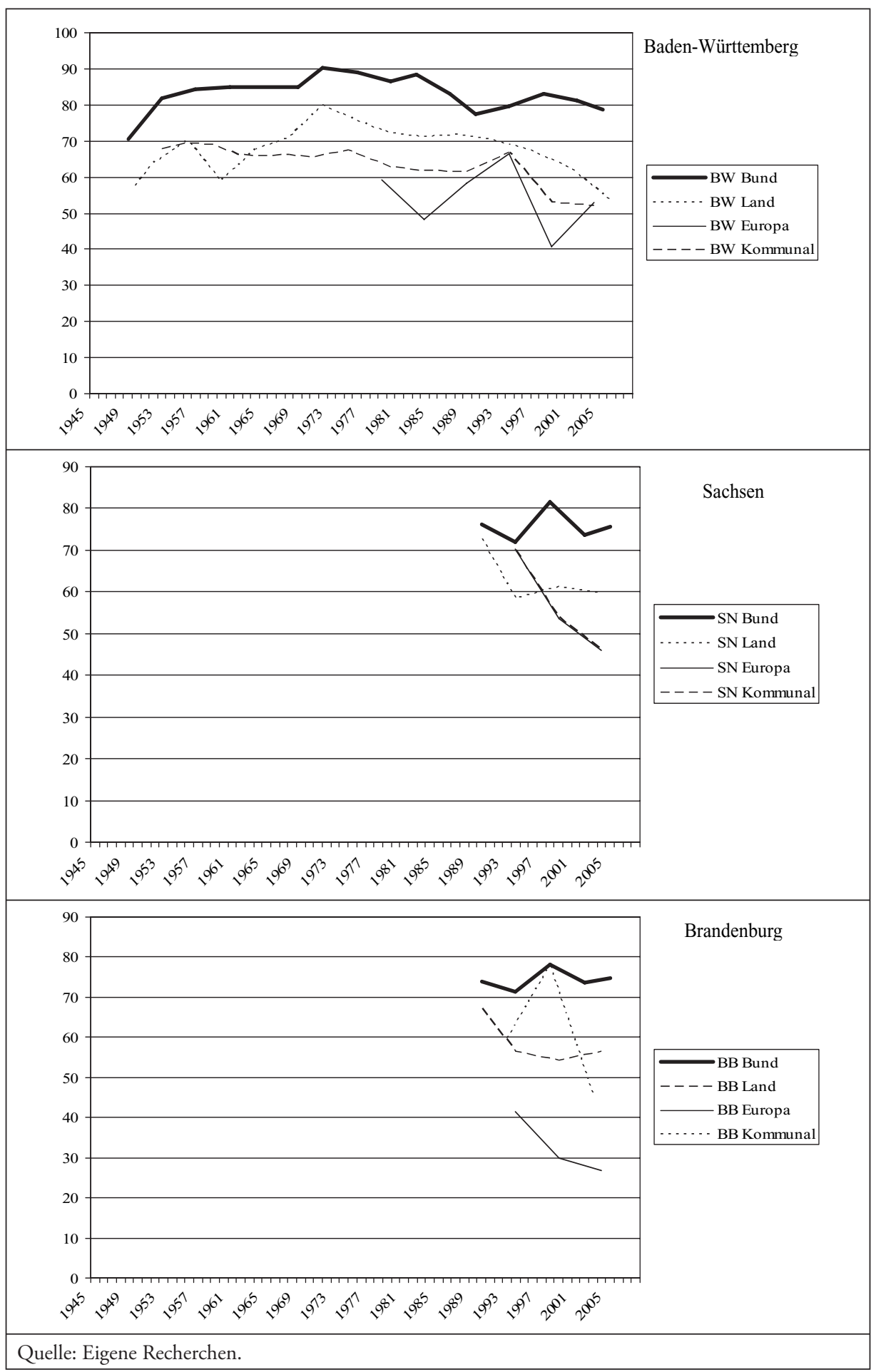


degradiert. ${ }^{24}$ Und falls Kommunal- und EP-Wahlen nicht am selben Tag stattfinden, wie im Saarland, in Rheinland-Pfalz, Sachsen, Sachsen-Anhalt, Thüringen oder Brandenburg, müssen letztere eindeutig auf den letzten Platz verwiesen werden. Ähnlich wie in anderen europäischen Staaten scheinen Wahlen zum EP damit von den Bürgern definitiv als „more second order than other elections" eingestuft zu werden. ${ }^{25}$

\section{Analysen}

Die folgenden Analysen basieren auf Aggregatdaten zu 114 Kommunalwahlen, die in den deutschen Flächenländern seit Gründung der Bundesrepublik Deutschland stattgefunden haben. In Hessen und Schleswig-Holstein fanden zwischen 1949 und 2008 je 14 Kommunalwahlen statt, in Niedersachsen 13, in Nordrhein-Westfalen, Rheinland-Pfalz und BadenWürttemberg je zwölf, in Bayern und dem Saarland je elf sowie in den neuen Bundesländern bislang je drei Wahlen (vgl. Tabelle 2).

\begin{tabular}{|l|c|c|c|c|}
\hline \multicolumn{5}{|l|}{ Tabelle 2: Datenübersicht } \\
\hline Bundesland & $\begin{array}{c}\text { Zahl der } \\
\text { Kommunalwahlen }\end{array}$ & von / bis & $\begin{array}{c}\text { Min./Max. } \\
\text { Wahlbeteiligung }\end{array}$ & $\begin{array}{c}\text { Min./Max. Abstand zur } \\
\text { nächsten BTW in Tagen }\end{array}$ \\
\hline SH & 14 & $1951-2003$ & $54,4-79,2$ & $163-2.194$ \\
NS & 13 & $1952-2006$ & $52,7-91,4$ & $0-1.113$ \\
NW & 12 & $1952-2004$ & $54,5-86,4$ & $0-1.110$ \\
HE & 14 & $1952-2006$ & $45,8-81,4$ & $28-1.294$ \\
RP & 12 & $1952-2004$ & $57,6-81,4$ & $112-1.197$ \\
BW & 12 & $1956-2004$ & $52,0-69,2$ & $105-1.064$ \\
BY & 11 & $1952-2008$ & $59,6-81,9$ & $161-1.294$ \\
SL & 11 & $1956-2004$ & $56,3-87,6$ & $126-1.197$ \\
BB & 3 & $1993-2003$ & $46,0-77,9$ & $0-570$ \\
MV & 3 & $1994-2004$ & $44,9-65,7$ & $126-1.197$ \\
SN & 3 & $1994-2004$ & $46,1-70,1$ & $126-1.197$ \\
SA & 3 & $1994-2004$ & $42,1-66,2$ & $126-1.197$ \\
TH & 3 & $1994-2004$ & $50,6-72,3$ & $126-1.197$ \\
\hline Gesamt & 114 & $1951-2008$ & $42,1-91,4$ & $0-1.294$ \\
\hline Quelle: Eigene Recherchen.
\end{tabular}

24 Ausnahmen hiervon sind Bayern, das Saarland und Schleswig-Holstein, in denen die Beteiligung an Landtags- und Kommunalwahlen nahezu identisch ist.

25 Vgl. beispielsweise zu Großbritannien Anthony Heath / Iain McLean / Bridget Taylor / John Curtice, Between First and Second Order: A Comparison of Voting Behavior in European and Local Elections in Britain, in: European Journal of Political Research, 35. Jg. (1999), H. 3, S. 389 $-414$. 
Die maximale Beteiligung betrug 91,4 Prozent bei der Kommunalwahl 1976 in Niedersachsen. Am geringsten war die Beteiligung mit 42,1 Prozent in Sachsen-Anhalt im Jahr 2004. Nur dreimal in der Geschichte der Bundesrepublik fielen Kommunalwahlen mit einem Bundestagswahltermin zusammen (Niedersachsen 1976, Nordrhein-Westfalen 1994 , Brandenburg 1994). Lässt man diese Wahlen außer Betracht, betrug der minimale Abstand zur nächsten Bundestagswahl 28 Tage (1972 in Hessen und Niedersachsen). Am weitesten von der nächsten Bundestagswahl entfernt fanden die Kommunalwahlen in Bayern 1966 und in Hessen 1977 statt (jeweils 1.294 Tage). Diese beiden Wahlen folgten innerhalb von sechs Monaten auf die vorhergehende Bundestagswahl.

Die erste graphische Analyse der Beteiligungsdaten - nach Wahlzyklen aufgeschlüsselt - zeigt keine systematischen Beteiligungsveränderungen kurz vor (und nach) den Bundestagswahljahren (vgl. Abbildung 4). Nur einige Ausreißer nach oben fallen auf, bei denen es sich um jene Wahlen handelt, die zusammen mit den Bundestagswahlen stattgefunden haben. Allerdings könnten vorhandene Zykluseffekte in der Graphik beispielsweise von den oben beschriebenen Beteiligungsdifferenzen zwischen den Bundesländern verdeckt werden. Um die Existenz von Zyklus- und Koppelungseffekte zu überprüfen, sind folglich differenziertere statistische Analysen notwendig, bei denen verschiedene Kontrollvariablen berücksichtigt werden.

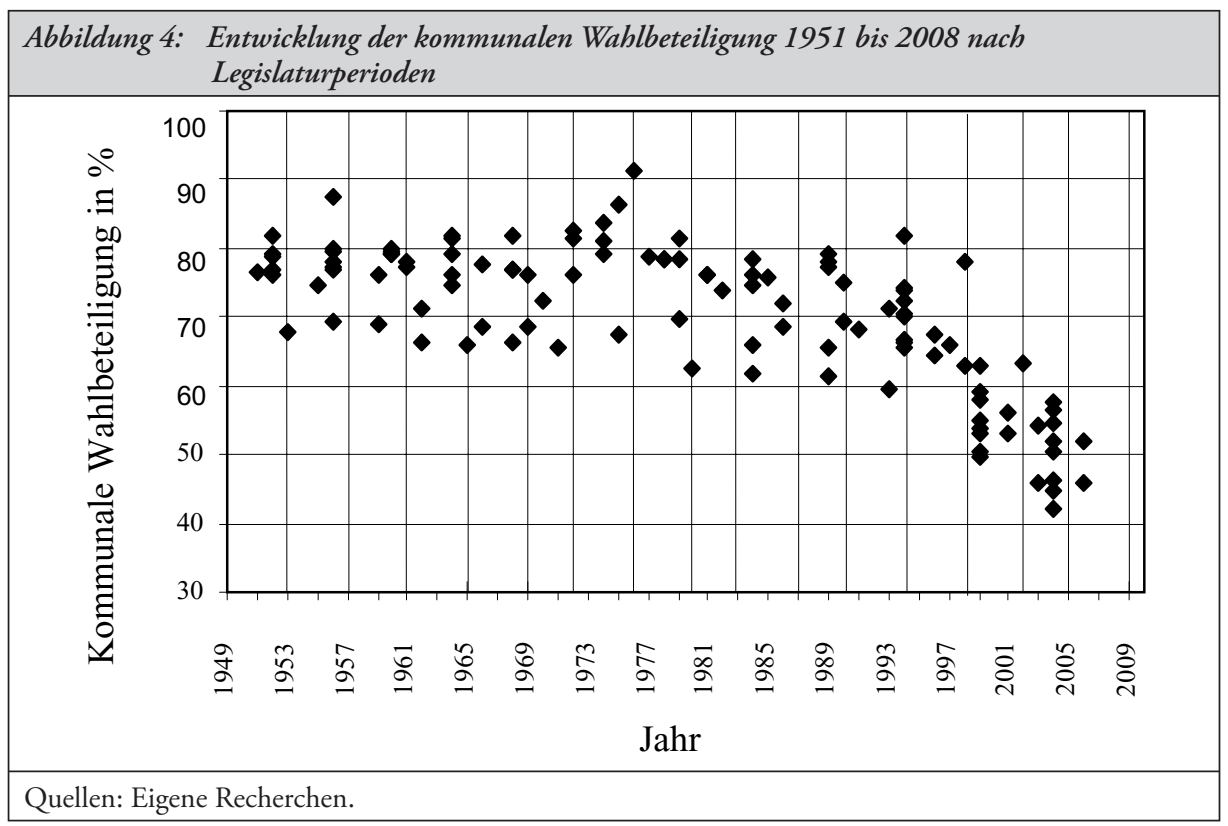

Die folgenden multivariaten Regressionsmodelle enthalten, erstens, als unabhängige Variable den zeitlichen Abstand der einzelnen Kommunalwahlen zur folgenden Hauptwahl (in Tagen) ${ }^{26}$, um die Existenz von Wahlzykluseffekten zu prüfen. Zweitens wird in Form einer

26 Um mögliche zyklische Abhängigkeiten zu prüfen, wird die Anzahl der Tage vor der nächsten Bundestagswahl außerdem als Quadratterm in die Kontrollgleichungen aufgenommen. 
Dummyvariable kontrolliert, ob die Koppelung mit einer EP-Wahl zu einer signifikant höheren Kommunalwahlbeteiligung führt. Der starke Rückgang der kommunalen Wahlbeteiligung, der unter anderem auf gesellschaftlich-politische Modernisierungsprozesse (Auflösung traditioneller kultureller Milieus und sozialer Klassen; Säkularisierung und Individualisierung der Gesellschaft; zunehmende Mobilität der Gesellschaftsmitglieder) zurückgeführt wird ${ }^{27}$, legt es zudem nahe, als Kontrollvariable das Jahr der jeweiligen Wahl in der Regression zu berücksichtigen, das sich negativ auf die Beteiligung auswirken sollte. Die deutlichen Beteiligungsunterschiede zwischen den Bundesländern werden wegen der beschränkten Fallzahl nicht mit Hilfe einzelner Länderdummyvariablen kontrolliert, sondern lediglich mit Hilfe einer Unterscheidung zwischen alten und neuen Bundesländern (Ost/West), da die Beteiligungsunterschiede hier am deutlichsten erkennbar sind. Ergänzend wird lediglich eine Kontrollvariable für Baden-Württemberg in die Regressionsmodelle aufgenommen, weil die Beteiligung an Kommunalwahlen dort signifikant niedriger ist als in allen anderen westlichen Bundesländern. Die folgenden Analysen werden zunächst für alle Wahlen seit 1951 durchgeführt. Anschließend werden die „Ausreißer“-Wahlen eliminiert und die Analysen getrennt für die Zeiträume von 1951 bis 1980 sowie von 1981 bis 2008 berechnet.

Am stärksten wird die Höhe der Kommunalwahlbeteiligung von den Prozessen bestimmt, die dem generellen Rückgang der Beteiligung in den letzten 20 Jahren zugrunde liegen, die hier aber nicht näher untersucht werden (vgl. Tabelle 3 bis 5). Über alle Jahre gerechnet nimmt die Beteiligung an Wahlen zu den lokalen Vertretungskörperschaften pro Jahr um etwa 0,4 Prozentpunkte ab. Über zehn Jahre gerechnet macht dies vier Prozentpunkte aus, über 60 Jahre 24 Prozentpunkte. Wählt man für die Analysen lediglich die Wahlen seit 1980 aus, beträgt der durchschnittliche Rückgang der Wahlbeteiligung sogar etwa ein Prozentpunkt pro Jahr. Ebenfalls deutlich erkennbar sind Effekte, die auf die kulturellen und/ oder institutionellen Differenzen zwischen den einzelnen Bundesländern zurückzuführen sind. Alle Modelle zeigen eine neun bis elf Prozentpunkte höhere Kommunalwahlbeteiligung in den alten Bundesländern mit Ausnahme von Baden-Württemberg. Dort liegt die Beteiligung nur knapp über den Werten für Ostdeutschland.

Der zeitliche Abstand zur nächsten Bundestagswahl wirkt sich demgegenüber deutlich schwächer auf die Höhe der kommunalen Wahlbeteiligung aus. Die Effekte sind signifikant, aber nicht besonders stark. Mit jedem Tag, den eine Kommunalwahl weiter von der nächsten Bundestagswahl entfernt liegt, nimmt die Beteiligung um durchschnittlich 0,006 Prozentpunkte ab. Liegt also eine Kommunalwahl ein Jahr vor der nächsten Bundestagswahl, ist die Beteiligung unter Kontrolle der anderen genannten Aspekte durchschnittlich 2,2 Prozentpunkte niedriger, als wenn die Wahl zusammen mit der Bundestagswahl stattfinden würde. In der Mitte der Wahlperiode beträgt die Differenz 4,4 Prozentpunkte, und drei Jahre vor der nächsten Bundestagswahl liegt die Quote 6,6 Prozentpunkte unter der Bundestagswahlbeteiligung. Selbst wenn die Wahlen ohne die „Ausreißer-Werte“ analysiert werden (ohne Kombiwahlen), bleibt der lineare Effekt signifikant.

27 Vgl. Markus Steinbrecher / Sandra Huber / Hans Rattinger, Turnout in Germany. Citizen Participation in State, Federal, and European Elections since 1979, Baden-Baden 2007, S. 4 f. 


\begin{tabular}{|c|c|c|c|c|}
\hline & $\begin{array}{c}\text { 1951-2008 } \\
\text { alle Wahlen } \\
\text { b/beta }\end{array}$ & $\begin{array}{c}1951-2008 \\
\text { ohne Kombiwahlen } \\
\text { b/beta }\end{array}$ & $\begin{array}{c}1951-1980 \\
\text { ohne Kombiwahlen } \\
\text { b/beta }\end{array}$ & $\begin{array}{c}\text { 1980-2008 } \\
\text { ohne Kombiwahlen } \\
\text { b/beta }\end{array}$ \\
\hline Konstante & 849 & 895 & 158 & 2.067 \\
\hline $\begin{array}{l}\text { Abstand vor } \\
\text { BTW (Tage) }\end{array}$ & $-0,006 /-0,2$ & $-0,004 /-0,1$ & $-0,003 /-0,2$ & $-0,006 /-0,2$ \\
\hline Abstand $^{2}$ & - & - & - & - \\
\hline $\begin{array}{l}\text { EP-Wahl } \\
\text { zeitgleich }\end{array}$ & $3,4 / 0,1$ & $5,0 / 0,2$ & ns & $4,7 / 0,2$ \\
\hline Jahr der Wahl & $-0,4 /-0,6$ & $-0,4 /-0,7$ & ns & $-1,0 /-0,7$ \\
\hline Ost/West & $10,0 / 0,3$ & $11,7 / 0,4$ & - & $8,7 / 0,4$ \\
\hline $\mathrm{BW}$ & $-9,7 /-0,3$ & $-9,3 /-0,3$ & $-11,3 /-0,7$ & $-8,1 /-0,2$ \\
\hline Adj. $R^{2}$ & 0,67 & 0,73 & 0,57 & 0,81 \\
\hline $\mathrm{N}$ & 113 & 110 & 53 & 57 \\
\hline
\end{tabular}

Anmerkungen: $\mathrm{ns}=$ nicht signifikant. Die b-Werte bedeuten, dass sich die lokale Wahlbeteiligung um $\mathrm{b}$ Einheiten verändert, wenn sich die jeweilige unabhängige Variable um eine Einheit verändert. Die betaWerte sind standardisierte Regressionskoeffizienten. Sie ermöglichen den Vergleich der Effekte zwischen den verschiedenen unabhängigen Variablen, unabhängig von der jeweiligen Skalierung.

Die Kommunalwahl in Nordrhein-Westfalen 1975, die zeitgleich mit der Landtagswahl stattfand, wurde von den Analysen als Ausreißerwahl ausgeschlossen. „Ohne Kombiwahlen“ bedeutet, dass die Modelle keine Kommunalwahlen enthalten, die zeitgleich mit Bundestagswahlen stattgefunden haben. Sie sind somit strengere Tests der Hypothesen.

Quelle: Eigene Recherchen bei den Statistischen Landesämtern.

\section{Tabelle 4: Kommunale Wablbeteiligung und zyklische Haupt-Nebenwabl-Effekte}

\begin{tabular}{|c|c|c|c|c|}
\hline & $\begin{array}{c}\text { 1951-2008 } \\
\text { alle Wahlen } \\
\text { b/beta }\end{array}$ & $\begin{array}{c}1951-2008 \\
\text { ohne Kombiwahlen } \\
\text { b/beta }\end{array}$ & $\begin{array}{c}1951-1980 \\
\text { ohne Kombiwahlen } \\
\text { b/beta } \\
\end{array}$ & $\begin{array}{c}\text { 1981-2008 } \\
\text { ohne Kombiwahlen } \\
\text { b/beta }\end{array}$ \\
\hline Konstante & 860 & 895 & 103 & 2.084 \\
\hline $\begin{array}{l}\text { Abstand vor } \\
\text { BTW (Tage) }\end{array}$ & $-0,02 /-0,7$ & ns & $\mathrm{ns}$ & $-0,014 /-0,5$ \\
\hline Abstand ${ }^{2}$ & $1,022^{-5} / 0,5$ & ns & ns & ns \\
\hline $\begin{array}{l}\text { EP-Wahl } \\
\text { zeitgleich }\end{array}$ & $3,4 / 0,1$ & $5,0 / 0,2$ & $\mathrm{~ns}$ & $4,3 / 0,2$ \\
\hline Jahr der Wahl & $-0,4 /-0,6$ & $-0,4 /-0,7$ & ns & $-1,0 /-0,7$ \\
\hline Ost/West & $10,6 / 0,3$ & $11,7 / 0,4$ & - & 8,8 / 0,4 \\
\hline BW & $-9,5 /-0,3$ & $-9,3 /-0,3$ & $-11,6 /-0,8$ & $-8,1 /-0,2$ \\
\hline Adj. $R^{2}$ & 0,68 & 0,72 & 0,58 & 0,81 \\
\hline $\mathrm{N}$ & 113 & 110 & 53 & 57 \\
\hline
\end{tabular}

Anmerkungen: $\mathrm{ns}=$ nicht signifikant. Die b-Werte bedeuten, dass sich die lokale Wahlbeteiligung um $\mathrm{b}$ Einheiten verändert, wenn sich die jeweilige unabhängige Variable um eine Einheit verändert. Die betaWerte sind standardisierte Regressionskoeffizienten. Sie ermöglichen den Vergleich der Effekte zwischen den verschiedenen unabhängigen Variablen, unabhängig von der jeweiligen Skalierung.

Die Kommunalwahl in Nordrhein-Westfalen 1975, die zeitgleich mit der Landtagswahl stattfand, wurde von den Analysen als Ausreißerwahl ausgeschlossen. „Ohne Kombiwahlen“ bedeutet, dass die Modelle keine Kommunalwahlen enthalten, die zeitgleich mit Bundestagswahlen stattgefunden haben. Sie sind somit strengere Tests der Hypothesen.

Quelle: Eigene Recherchen bei den Statistischen Landesämtern. 
Die lineare Haupt-Nebenwahl-These ist damit für Kommunalwahlen in der Bundesrepublik Deutschland bestätigt. Sie gewinnt an Kraft, wenn die Analysen für die ersten 30 Jahre der Republik (bis 1980) und die Zeit des verstärkten Medieneinflusses auf Politik von 1981 bis heute getrennt durchgeführt werden. Der lineare Haupt-Nebenwahl-Effekt ist bereits in den Jahren bis 1980 erkennbar (neben dem damals schon offensichtlichen und starken „Baden-Württemberg Malus“). Er wird in der zweiten Phase von 1980 bis heute jedoch deutlich stärker. Ob dies mit dem Rückgang der Wählerbindungen oder der verstärkten Medienmobilisierung im Zusammenhang mit Bundestagswahlkämpfen zusammenhängt, lässt sich an dieser Stelle nicht klären. Aber offensichtlich ist der Effekt keine „Erfindung“ der letzten Jahre.

\begin{tabular}{|c|c|c|c|c|c|c|c|c|}
\hline & \multicolumn{4}{|c|}{$\begin{array}{l}\text { Bundesländer mit traditionell über- } \\
\text { durchschnittlicher Mobilisierung bei } \\
\text { Kommunalwahlen } \\
\text { NS, SL, HE, RP, BAY }\end{array}$} & \multicolumn{4}{|c|}{$\begin{array}{c}\text { Bundesländer mit traditionell unter- } \\
\text { durchschnittlicher Mobilisierung bei } \\
\text { Kommunalwahlen } \\
\text { BW, BB, MV, SN, SA, TH }\end{array}$} \\
\hline & \multicolumn{2}{|c|}{ Linear } & \multicolumn{2}{|c|}{ Zyklisch } & \multicolumn{2}{|c|}{ Linear } & \multicolumn{2}{|c|}{ Zyklisch } \\
\hline & $\begin{array}{l}1951- \\
2008 \\
\text { b/beta }\end{array}$ & $\begin{array}{l}1980- \\
2008 \\
\text { b/beta }\end{array}$ & $\begin{array}{l}1951- \\
2008 \\
\text { b/beta }\end{array}$ & $\begin{array}{c}1980- \\
2008 \\
\text { b/beta }\end{array}$ & $\begin{array}{c}1951- \\
2008 \\
\text { b/beta }\end{array}$ & $\begin{array}{c}1980- \\
2008 \\
\text { b/beta }\end{array}$ & $\begin{array}{l}1951- \\
2008 \\
\text { b/beta }\end{array}$ & $\begin{array}{c}1980- \\
2008 \\
\text { b/beta }\end{array}$ \\
\hline Konstante & 953 & 2.096 & 950 & 2.057 & 1.047 & 2.550 & 938 & 1.696 \\
\hline $\begin{array}{l}\text { Abstand vor } \\
\text { BTW (Tage) }\end{array}$ & ns & $\begin{array}{c}-0,006 / \\
-0,2\end{array}$ & $\mathrm{~ns}$ & ns & $\begin{array}{c}-0,008 / \\
-0,3\end{array}$ & $\begin{array}{c}-0,007 / \\
-0,3\end{array}$ & $\begin{array}{c}-0,005 / \\
-2,2\end{array}$ & $\begin{array}{c}-0,063 / \\
-2,8\end{array}$ \\
\hline Abstand $^{2}$ & - & - & $\mathrm{ns}$ & ns & - & - & $\begin{array}{c}3,314^{-5} \\
/ 1,9\end{array}$ & $\begin{array}{c}4,044^{-5} \\
12,5\end{array}$ \\
\hline $\begin{array}{l}\text { EP-Wahl } \\
\text { zeitgleich }\end{array}$ & $\begin{array}{l}4,2 / \\
0,2\end{array}$ & $\begin{array}{l}3,0 / \\
0,2\end{array}$ & $\begin{array}{l}4,1 / \\
0,2\end{array}$ & $\begin{array}{l}4,1 / \\
0,2\end{array}$ & ns & $\begin{array}{l}5,2 / \\
0,2\end{array}$ & $\mathrm{~ns}$ & ns \\
\hline $\begin{array}{l}\text { Jahr der } \\
\text { Wahl }\end{array}$ & $\begin{array}{l}-0,4 / \\
-0,8\end{array}$ & $\begin{array}{l}-1,0 / \\
-0,9\end{array}$ & $\begin{array}{l}-0,4 / \\
-0,8\end{array}$ & $\begin{array}{l}-1,0 / \\
-0,9\end{array}$ & $\begin{array}{l}-0,5 / \\
-0,8\end{array}$ & $\begin{array}{l}-1,3 / \\
-0,8\end{array}$ & $\begin{array}{l}-0,4 / \\
-0,7\end{array}$ & $\begin{array}{l}-0,8 / \\
-0,5\end{array}$ \\
\hline Adj. $R^{2}$ & 0,63 & 0,83 & 0,63 & 0,84 & 0,59 & 0,72 & 0,73 & 0,87 \\
\hline $\mathrm{N}$ & 60 & 28 & 60 & 28 & 26 & 19 & 26 & 19 \\
\hline
\end{tabular}

Anmerkungen: $n s$ = nicht signifikant. Die b-Werte bedeuten, dass sich die lokale Wahlbeteiligung um $b$ Einheiten verändert, wenn sich die jeweilige unabhängige Variable um eine Einheit verändert. Die betaWerte sind standardisierte Regressionskoeffizienten. Sie ermöglichen den Vergleich der Effekte zwischen den verschiedenen unabhängigen Variablen, unabhängig von der jeweiligen Skalierung.

Die Modelle enthalten keine Kommunalwahlen, die zeitgleich mit einer Bundestags- oder Landtagswahl stattgefunden haben. Es handelt sich hierbei folglich um strengere Tests der Zyklushypothese.

Quelle: Eigene Recherchen bei den Statistischen Landesämtern.

Lässt sich alternativ eine zyklische Haupt-Nebenwahl-Beziehung bestätigen, wie sie für die Veränderung der Stimmenverhältnisse in Landtagswahlen häufig nachgewiesen wurde? Die Kurvilinearität der Beziehung wird lediglich bei der Analyse aller Wahlen signifikant. Das heißt, es sind die „Ausreißer“-Wahlen, die diesen zyklischen Beteiligungseffekt hervorrufen. Entfernt man diese Fälle - was zur korrekten Hypothesenprüfung notwendig ist -, verschwindet die zyklische Beziehung. Dies belegen auch die nahezu unveränderten Varianzaufklärungswerte (adj. $\mathrm{R}^{2}$ ) im Vergleich zwischen den Tabellen 3 und 4. Selbst bei Teilung des Untersuchungszeitraums in die Jahre vor und nach 1980 lässt sich keine signifikante kurvilineare Beziehung feststellen. Wahlkämpfe auf Bundesebene wirken demnach kaum 
über die Bundestagswahl hinaus mobilisierend auf andere Wahlen - wie auch immer die Wirkungsmechanismen im Einzelnen aussehen könnten.

Darüber hinaus wurden variierende Einflüsse des „politischen“ Kontextes in Abhängigkeit von der traditionellen Mobilisierung der Bürger bei Kommunalwahlen vermutet. Auf die deutlichen und dauerhaft erkennbaren Beteiligungsdifferenzen zwischen den Bundesländern wurde bereits weiter oben verwiesen. Für den Hypothesentest wurden die Bundesländer in zwei Gruppen unterteilt: Das Saarland, Niedersachsen, Hessen, Rheinland-Pfalz und Bayern werden der Gruppe mit einer traditionell eher starken Wählermobilisierung zugeordnet, Baden-Württemberg und die fünf ostdeutschen Bundesländer dagegen der Gruppe mit traditionell geringer kommunaler Beteiligung. Hier - so die theoretischen Überlegungen - müssten externe Einflüsse aufgrund von zeitnahen Bundestagswahlen stärker zu erkennen sein als in der ersten Gruppe, da ein größerer Anteil an „peripheren “Wählern den Kommunalwahlen traditionell fern bleibt. Die empirischen Befunde scheinen diese Überlegung zu bestätigen:

Bei traditionell starker Mobilisierung ist ein linearer Haupt-Nebenwahl-Effekt seit 1980 nur schwach erkennbar. Eine zyklische Beziehung kann überhaupt nicht nachgewiesen werden (vgl. Tabelle 5). Hiervon unterscheiden sich die Befunde in der „low mobilization“ Gruppe deutlich. Nicht nur der lineare Effekt trägt jetzt zur Varianzerklärung bei (sowohl für den gesamten Zeitraum als auch für die Jahre ab 1980). Vielmehr tritt nun auch ein zyklischer Haupt-Nebenwahl-Effekt deutlich zu Tage. Die beiden zyklischen Modelle in Tabelle 5 zeigen für Baden-Württemberg und die fünf Bundesländer in Ostdeutschland eine deutlich höhere Varianzaufklärung als die linearen Modelle. Das heißt, unter der Voraussetzung einer traditionell geringen Wahlbeteiligung sind mobilisierende Einflüsse durch die Bundestagswahlen deutlich erkennbar, und diese - das ist das Besondere - wirken offensichtlich nicht nur im Vorfeld der Wahlen, wenn Parteien und Massenmedien zu einem insgesamt ansteigenden Interesse der Bürger an Politik beitragen. Vielmehr hält dieser Mobilisierungsschub über die Wahl hinaus an.

Dieser Interaktionsbefund ist theoretisch plausibel, und die Stärke der Effekte in Tabelle 5 ist deutlich. Allerdings - so muss einschränkend gesagt werden - beruhen die Analysen nur auf einer vergleichsweise kleinen Anzahl von Fällen. Diese bilden nur zwei Wahlzyklen ab - die Jahre zwischen 1994 und 2002. Damit könnte ihre Aussagekraft unter Umständen eingeschränkt sein, denn zum einen handelte es sich 1994 - ähnlich wie 2009 - um ein „Superwahljahr“. Damit könnten die Beteiligungsquoten von 1994 „verzerrt“ sein. Zweitens könnte sich 1994 noch die „Vereinigungseuphorie“ in der Höhe der kommunalen Wahlbeteiligung widerspiegeln, zumal es sich in den meisten neuen Bundesländern um die ersten Kommunalwahlen mit neuer Kommunalverfassung handelte. Solche möglichen situationsbedingten Effekte sind vor allem problematisch, wenn die Fallzahl gering ist. Daher muss die hier aufgedeckte interaktive Beziehung zwischen kommunalen Wahlen und Bundestagswahlen in den nächsten Jahren sorgfältig weiter beobachtet werden.

Welche Auswirkungen hat abschließend die Koppelung kommunaler Wahlen mit Wahlen zum Europäischen Parlament für die Höhe der Wahlbeteiligung? Führt die Tatsache, dass der Wähler mit einem Wahlgang gleichzeitig zwei Entscheidungen treffen kann, zu einer verstärkten Bereitschaft, sich an der Wahl zu beteiligen? Die Ergebnisse lassen auf einen solchen Stimulierungseffekt schließen. Bis auf wenige Ausnahmen (die Analysen für den Zeitraum 1951 bis 1980, in dem nur zwei Kommunalwahlen mit EP-Wahlen gekoppelt waren) werden positive Effektkoeffizienzen ermittelt. Dabei variiert der durchschnittli- 
che Einfluss einer Koppelung unter Kontrolle der übrigen in den Modellen enthaltenen Faktoren zwischen drei und fünf Prozent, das heißt, wenn Kommunal- und EP-Wahlen am selben Tag stattfinden, liegt die Beteiligungsquote drei bis fünf Prozentpunkte höher als ohne Koppelung. Dieser Befund dürfte für die Praxis äußerst relevant sein, da eine Zusammenlegung der Termine folglich nicht nur mit geringeren Organisationskosten verbunden ist, sondern gleichzeitig zu einer stärkeren Mobilisierung führt.

\section{Zusammenfassung und Diskussion: Koppelung der Wahlen für mehr Beteiligung?}

Timing ist offensichtlich wichtig, wenn es um die Beteiligung an Kommunalwahlen geht. Dies haben die Analysen vor allem für die letzten Jahre gezeigt. Am höchsten ist die Beteiligung natürlich, wenn kommunale Wahlen zeitgleich mit Bundestagswahlen stattfinden. In den meisten Bundesländern sind solche Koppelungen mit Bundestagswahlen jedoch verboten, oder aber sie werden bewusst umgangen, um den subnationalen Wahlen eine eigene Qualität zu geben. Dennoch gilt: Je näher eine Kommunalwahl an einer Bundestagswahl liegt, desto höher ist die kommunale Wahlbeteiligung, auch wenn der Effekt schwach ist. Die Ursachen hierfür sind unklar. Die Vermutung liegt aber nahe, dass lokale Wahlen dann von der politischen Mobilisierung der nationalen Parteien und der Massenmedien in dieser Zeit und von den verstärkten Wahlkampfanstrengungen der lokalen Parteiorganisationen profitieren. Die höhere Beteiligung dürfte einerseits auf die Mobilisierung „peripherer“ Wähler zurückzuführen sein. Bürger mit weniger Interesse an Politik wenden sich während des Bundestagswahlkampfes stärker der Politik zu als sonst und finden unter Umständen auch Interesse an kommunalen Fragen. Andererseits werden im Laufe der Wahlkämpfe die „eigenen Reihen geschlossen“, das heißt im Vorfeld von Bundestagswahlen werden auch die eigenen Parteianhänger aktiviert, was sich wiederum in der Höhe der kommunalen Wahlbeteiligung bemerkbar machen müsste. Wichtig ist jedenfalls, dass die Kommunalwahl im Vorfeld der Bundestagswahl stattfindet. Zyklische Haupt-Nebenwahl-Effekte konnten nicht nachgewiesen werden. Kurz nach einer Bundestagswahl dürfte die kommunale Wahlbeteiligung ceteris paribus folglich am geringsten sein.

Die Koppelung von Kommunalwahlen mit EP-Wahlen scheint sich ebenfalls positiv auf die Höhe der Wahlbeteiligung auszuwirken. Dies war nicht unbedingt zu erwarten. Die Beschlüsse, die im Europäischen Parlament getroffen werden, sehen die Deutschen in der Regel als noch unwichtiger an als Entscheidungen der lokalen Vertretungskörperschaften. Daher könnten EP-Wahlen - ähnlich wie in Großbritannien - unter Umständen als „third order elections" interpretiert werden mit entsprechend demobilisierenden Folgen für die Kommunalwahlen. Dies ist aber nicht der Fall. Im Gegenteil, auch wenn die Effekte nicht hochsignifikant sind, scheint die Beteiligung an gekoppelten Kommunal- und EP-Wahlen um drei bis fünf Prozentpunkte höher zu liegen als bei getrennten Wahlen.

Sollten Kommunalwahlen zukünftig folglich zeitlich in die Nähe von Bundestagswahlen gerückt und an Wahlen zum Europäischen Parlament gekoppelt werden? Ein solcher Schritt käme einer vertikalen Angleichung von Wahlen gleich und dürfte ähnliche Folgen haben wie die Alternative einer horizontalen Vereinheitlichung der kommunalen Wahltermine, unabhängig vom Bundestagswahltermin (horizontale Gleichzeitigkeit). Heute finden die Wahlen zu den lokalen Vertretungskörperschaften in jedem Bundesland zu einem jeweils anderen Zeitpunkt statt. Lediglich in einigen Bundesländern liegen die Kommunalwahlen 
bewusst auf dem Tag der EP-Wahl, wodurch es bereits zu einer gewissen horizontalen Gleichzeitigkeit kommt. Dennoch dominiert noch immer die konsekutive Abfolge. Die Literatur geht bei beiden Strategien - der vertikalen und der horizontalen Simultaneität von einem Verlust von Eigenständigkeit der subnationalen Wahlen aus. ${ }^{28}$ Eine vertikale ebenso wie eine horizontale Vereinheitlichung hätten sicherlich größere Aufmerksamkeit für die Kommunalwahlen und damit eine höhere Beteiligung zur Folge. Diese wäre vermutlich aber mit einem wesentlich stärkeren bundespolitischen Einfluss auf die Wahl und einer größeren Vereinheitlichung der Wahlergebnisse erkauft: Den Wahlen käme mehr Signalwirkung für die nationale Ebene zu. Parteien und Medien würden folglich aktiver. Der Wahlkampf wäre bundesweit spürbar und in weiten Teilen einheitlich. Nationale Themen würden wahrscheinlich die Wahlentscheidung erheblich beeinflussen. Bei horizontaler Simultaneität der Kommunalwahlen in der Mitte des Wahlzyklus müsste bundesweit mit einem deutlichen Stimmenverlust für die Regierungsparteien gerechnet werden. Umgekehrt dürften die Stimmenverluste für die Regierungsparteien bei den Kommunalwahlen bundesweit deutlich geringer sein, je näher die Wahlen an der nächsten Bundestagswahl liegen. Regionale Unterschiede würden über die Zeit vermutlich verschwinden, während bundeseinheitliche Muster auf der lokalen Ebene erwartet werden.

Diese Argumente wurden hinsichtlich der Frage der Zusammenlegung von Landtagswahlen bereits umfänglich diskutiert. Klaus Detterbeck schlussfolgerte: „Der ,Preis’, der durch einen Sieg bei simultanen Regionalwahlen zu holen ist, ist höher, als dies bei konsekutiven Wahlen der Fall ist. Es wird schlicht um mehr gehen bei diesen ,Superwahlen' ."29 Bezogen auf die Kommunalwahlen in Deutschland ist dieser Schluss jedoch nicht zwingend, da die genannten Folgen einer Vereinheitlichung des Wahltermins nicht zwangsläufig zu erwarten sind. Erstens hat der Ausgang der Kommunalwahlen im politischen System der Bundesrepublik Deutschland keinen institutionalisierten Einfluss auf die Bundespolitik, wie dies bei Landtagswahlen anzutreffen ist. Ergebnisse von Kommunalwahlen könnten höchstens als symbolisches Signal nach Berlin interpretiert werden. Dem stehen jedoch zwei weitere Gründe entgegen, die die Unabhängigkeit der Kommunalwahlen von der Bundespolitik stärken könnten. Zum einen sind die in den Bundesländern institutionalisierten Kommunalwahlsysteme deutlich stärker personenorientiert als die Landtags- oder Bundestagswahlsysteme. ${ }^{30}$ Die Möglichkeiten des Kumulierens und Panaschierens von Stimmen, die mittlerweile in zahlreichen Bundesländern gegeben sind, schwächen die lokalen Parteien und geben den Kandidaten gegenüber ihren Parteien ein stärkeres Gewicht. Dies wiederum hat zur Folge, dass die parteipolitische Durchdringung der Kommunalwahlen in der Bundesrepublik Deutschland nach der Änderung der kommunalen Wahlsysteme in den letzten Jahren schwieriger geworden ist. Zum anderen finden wir mittlerweile - mit leichten Unterschieden zwischen den Bundesländern - auf der lokalen Ebene häufig ein von der Bundespolitik abweichendes Parteiensystem. Dies gilt für zahlreiche Kommunen in den alten Bundesländern, in denen Freie Wählergemeinschaften stark sind, wie zum Beispiel in Baden-Württemberg oder Bayern, zunehmend aber auch in anderen Bundesländern. Auch diese Unterschiede im Parteienangebot vor Ort dürften die bundesweite Verein-

28 Vgl. Klaus Detterbeck, a.a.O. (Fn. 18), S. 25 ff. mit Bezug zu Landtagswahlen. Analog kann die Diskussion auf Kommunalwahlen übertragen werden.

29 Klaus Detterbeck, a.a.O. (Fn. 18), S. 29.

30 Vgl. Angelika Vetter, a.a.O. (Fn. 20). 
heitlichung der Kommunalwahlen erschweren. Solange diese lokalen Besonderheiten zugelassen werden und institutionell abgesichert sind - zum Beispiel durch den Wegfall der Fünfprozentklausel bei Kommunalwahlen oder das stärker personalisierte Wahlrecht -, ist von einer bundespolitischen Durchdringung der Kommunalwahlen nicht zwangsweise auszugehen.

Die Nachteile einer horizontalen Vereinheitlichung der Kommunalwahlen sind damit nicht so stark zu gewichten wie im Fall einer Vereinheitlichung der Landtagswahlen. Umgekehrt könnten die Vorteile einer stärkeren bundesweiten Aufmerksamkeit genutzt werden. Sie kämen nicht nur der Wahlbeteiligung zugute. Vielmehr bekäme die Kommunalpolitik als dritte Ebene im politischen Mehrebenensystem insgesamt mehr Bedeutung, als ihr heute zuteil wird. Die Kommunen werden von den Politikern in Bund und Ländern, den Medien, aber auch von den Bürgern kaum wahrgenommen. Dies erstaunt angesichts der Tatsache, dass in der Bundesrepublik Deutschland die kommunale Selbstverwaltung nicht nur in Art. 28 Abs. 2 GG explizit garantiert ist. Vielmehr sind die Kommunen verantwortlich für die öffentliche Daseinsvorsorge, für die Bereitstellung und Versorgung der Bürger mit zentralen Gütern des täglichen Lebens und Zusammenlebens (Wasser- und Energieversorgung, Umweltschutz, Wirtschaftsförderung, Bauvorhaben, Ausländerintegration, Kinderbetreuung, sozialer Wohnungsbau etc.). Etwa ein Viertel aller Staatsausgaben werden über die örtlichen Verwaltungen getätigt, was die Bedeutung der Kommunen im gesamten Staatsgefüge verdeutlicht. ${ }^{31}$ Mehr Aufmerksamkeit der Politik und der Medien für die Kommunen wäre damit nicht nur vor dem Hintergrund einer höheren Wahlbeteiligung wünschenswert. Innerhalb des bundesdeutschen Mehrebenensystems mit seiner zu Unrecht fast ausschließlichen Fokussierung auf die Bundes- und Landespolitik käme sie einer stärkeren Anerkennung der lokalen Politik für das tägliche Lebensumfeld der Bürger gleich.

31 Vgl. Gerd Schmidt-Eichstaedt, Autonomie und Regelung von oben. Zum Verhältnis von kommunaler Eigenverantwortung und fremdbestimmter Eingrenzung durch Bundes- und Landesrecht sowie durch Normen der Europäischen Union, in: Hellmut Wollmann / Roland Roth (Hrsg.), Kommunalpolitik. Politisches Handeln in den Gemeinden, Opladen 1999, S. 323 337; Angelika Vetter, Changes in German Local Autonomy, in: Edward Page / Michael Goldsmith (Hrsg.), Local Autonomy in Europe (im Erscheinen).

\title{
Verfassungswandel in Großbritannien unter Labour - veränderte Handlungsspielräume des Parlaments
}

\author{
Merten Haring
}

Am 1. Mai 1997 gelang es Labour unter Tony Blair, die Wahlen zum Unterhaus zu gewinnen, eine Mehrheit von 118 Sitzen zu erzielen und dadurch mit 448 Abgeordneten die größte Unterhausfraktion in der Geschichte der Partei zu bilden und die Konservativen nach 18 Jahren als Regierungspartei abzulösen. Blair blieb, wie noch kein Labour-Chef vor ihm, drei Wahlen hintereinander ungeschlagen und amtierte zehn Jahre lang als Premierminister - auch dies ein Rekord unter Labour-Amtsinhabern. In seine Regierungszeit fallen 